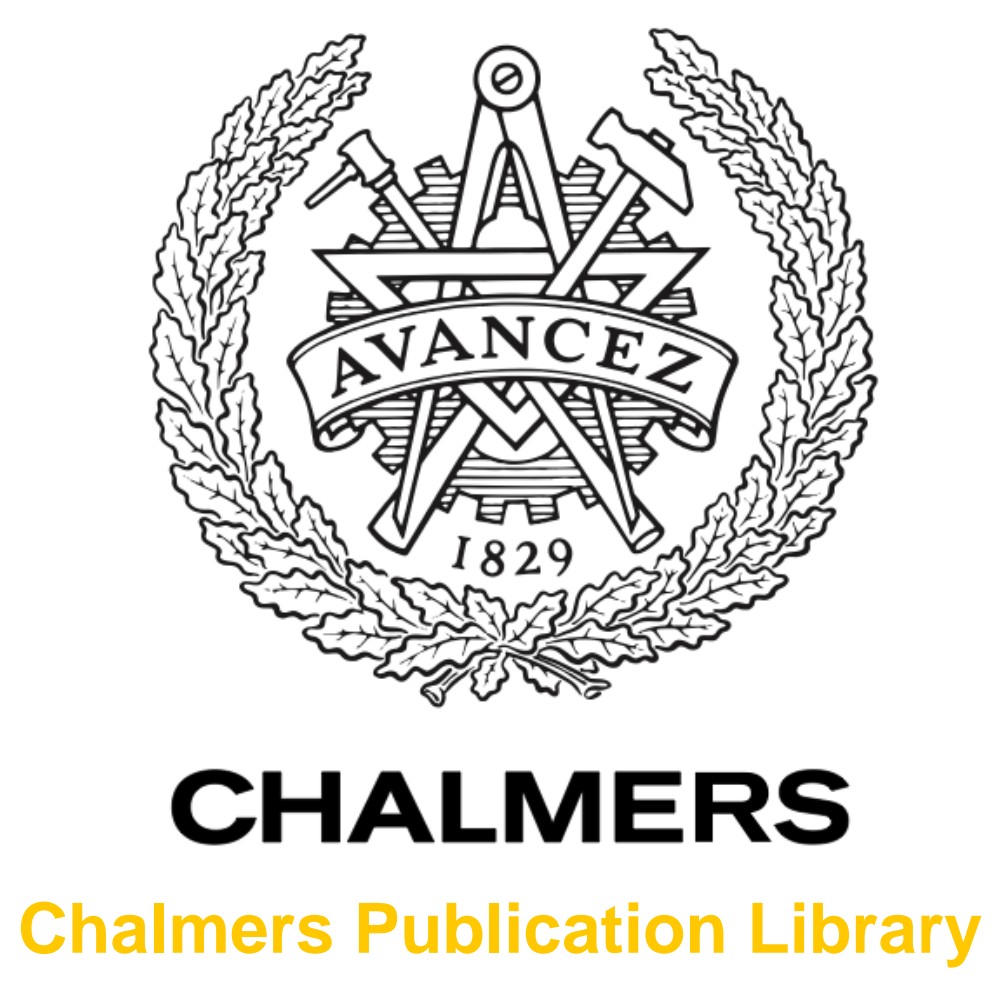

\title{
Throughput Modeling and Measurement in an Isotropic-Scattering Reverberation Chamber
}

This document has been downloaded from Chalmers Publication Library $(\mathrm{CPL})$. It is the author's version of a work that was accepted for publication in:

leee Transactions on Antennas and Propagation (ISSN: 0018-926X)

Citation for the published paper:

Chen, X. (2014) "Throughput Modeling and Measurement in an Isotropic-Scattering Reverberation Chamber". Ieee Transactions on Antennas and Propagation, vol. 62(4), pp. 2130-2139.

http://dx.doi.org/10.1109/tap.2014.2301850

Downloaded from: http://publications.lib.chalmers.se/publication/199237

Notice: Changes introduced as a result of publishing processes such as copy-editing and formatting may not be reflected in this document. For a definitive version of this work, please refer to the published source. Please note that access to the published version might require a subscription. 


\title{
Throughput Modeling and Measurement in an Isotropic-Scattering Reverberation Chamber
}

\author{
Xiaoming Chen
}

\begin{abstract}
This paper presents simple throughput models for multiple-input multiple-output orthogonal frequency division multiplexing (MIMO-OFDM) systems with hybrid automatic repeat request (HARQ). In particular, we consider the long term evolution (LTE) systems. Antenna correlations and power imbalances are implicitly included in the models. In order to explicitly characterize the antenna effects on throughput, we resort to the spatial multiplexing (SM) efficiency. The SM efficiencies of a MIMO antenna with zero forcing (ZF), minimum mean square error (MMSE), and maximum likelihood (ML) receivers are studied via numerical simulations using the throughput models. To verify the throughput models, the throughputs of two LTE terminals (including antenna correlations and power imbalances) are measured in a reverberation chamber (RC). Good agreements between the measured and modeled throughputs are observed.
\end{abstract}

Index Terms-Multiple-input multiple-output (MIMO), reverberation chamber (RC), throughput.

\section{INTRODUCTION}

$\mathbf{R}$ EVERBERATION chambers (RCs) have been used for various over-the-air (OTA) tests over the past decade [1]-[8]. Very recently, it has been used for measuring throughputs of long term evolution (LTE) systems [6]-[8]. Besides the $\mathrm{RC}$ (with/without fading emulator), there exist five more OTA testing systems for evaluating MIMO terminals: ${ }^{1}$ the anechoic chamber (AC) and fading emulator based multi-probe system (anechoic chamber single- and multiple-cluster), the decomposition method, and the two-stage OTA system (two-stage method radiated and conducted) (cf. [1] and reference therein). All the different types of OTA systems have pros and cons: the two-stage OTA system can readily implement various channel models, yet it is constrained by the availability of external antenna ports on the DUT and the requirement that complex radiation pattern must be nonintrusive with special chipset functions; the multi-probe system can flexibly emulate channels with different angular distributions, yet the cost is high due to the need of an $\mathrm{AC}$ and possibly multiple fading

\footnotetext{
Manuscript received September 24, 2013; revised November 13, 2013; accepted January 17, 2014. Date of publication January 21, 2014; date of current version April 03, 2014.

The author is with the Department of Signals and Systems, Chalmers University of Technology, 41296 Gothenburg, Sweden (e-mail: xiaoming. chen@chalmers.se).

Color versions of one or more of the figures in this paper are available online at http://ieeexplore.ieee.org.

Digital Object Identifier 10.1109/TAP.2014.2301850

${ }^{1}$ According to the 3GPP specification group RAN4 TR37.977.
}

emulators; ${ }^{2}$ the $\mathrm{RC}$ is limited to channels with the isotropic angular distribution, ${ }^{3}$ 0-dB cross polarization ratio (XPR), and limited temporal characteristics (unless a fading emulator is used). Further comparisons of different OTA testing systems are out of the scope of this work. The common advantages of OTA testing systems over field tests (i.e., real-life measurements) are repeatability and measurement efficiency.

Despite of the convenience of the OTA testing, there is still a need to develop simple models with reasonable accuracies. The reasons are multifold: a sound model allows one to double check the testing results and therefore helps eliminate potential systematic errors in measurements; the model can be used to complement measurements with arbitrary antenna effects (e.g., antenna correlation and power imbalance) and certain spatial equalization algorithms; the model enables more insight into the system under test. Currently, there is a great interest in conducting throughput measurements of LTE devices. As a result, throughput models have been developed in [7] and [8]. Nevertheless, the throughput model in [7] is only for single-input multiple-output (SIMO) systems; the throughput model in [8] is limited to open-loop MIMO systems with zero forcing (ZF) receivers; and both [7] and [8] focus on antenna effects at the receive side, whereas the transmit antennas are assumed to be uncorrelated.

In this paper, we extend the throughput model to including minimum mean square error (MMSE) and maximum likelihood (ML) receivers, and closed-loop configuration that are supported by LTE systems. Apart from studying antenna effects at the receive side, we also investigate antenna effects at the transmit side. The modeled throughputs are compared with the throughputs of two LTE terminals measured in an RC. Instead of focusing on the power imbalance in throughput measurements as in [7] and [8], this work contains throughput measurements in the presence of both power imbalance and antenna correlation. In addition, this work reexamines the effective frequency diversity order of the orthogonal frequency division multiplexing (OFDM) system with spatial diversity or multiplexing, resulting in better agreements of the modeled and measured throughputs for the spatial multiplexing case, as opposed to [8].

The antenna effects are included implicitly in the throughput models. To explicitly characterize the antenna effects, we resort

\footnotetext{
${ }^{2}$ There are proposal of a two-dimensional (2D) channel emulation implementation without the use of costly channel emulators. There are also proposal of a $3 \mathrm{D}$ channel emulation using only one channel emulator.

${ }^{3}$ Note that the RC is not instantaneously isotropic, but statistically isotropic. In this work, we simply refer to the statistically isotropic property as the isotropic property.
} 
to the spatial multiplexing (SM) efficiency [10] (to be defined in Section III). We compare the simulated SM efficiencies of the $\mathrm{ZF}$, MMSE, and ML receivers with the measured one of an LTE system in an RC. It is shown that the throughput model with the $M L$ receiver tends to underestimate the throughput degradation due to the antenna effects, while the ZF- and MMSE-receiver models agree well with the measured SM efficiency of the LTE device.

\section{Notations}

Throughout this paper, ${ }^{*},{ }^{T}$, and ${ }^{H}$ denote complex conjugate, transpose, and Hermitian operators, respectively. Lowercase letter $(x)$, lowercase bold letter $(\mathbf{x})$, and uppercase bold letter $(\mathbf{X})$ represent scalar, column vector, and matrix, respectively. $\|\mathbf{x}\|_{2}$ denotes the 2-norm of $\mathbf{x} . x_{i}$ and $\mathbf{x}_{i}$ denote the $i$ th element and column of $\mathbf{x}$ and $\mathbf{X}$, respectively. $[\mathbf{X}]_{i, i}$ denotes the $i$ th diagonal element of $\mathbf{X}$. Throughout this paper, $E$ denotes the expectation and $\mathbf{I}$ represents the identity matrix.

\section{THROUGHPUT MODELS}

As mentioned in the introduction, the goals of the throughput modeling are to provide a convenient tool to double check and complement throughput measurements and to enable more insight into the system under test. Therefore, the developed throughput models should be simple (i.e., with low computational complexity) while providing sufficient accuracy. To that end, the threshold receiver model [9] (or equivalently the outage theorem [11]) is used to get rid of the time-consuming channel coding in simulations.

\section{A. Throughput Model for SISO System}

Due to the heavy computational burden of implementing the channel code (e.g., Turbo code in LTE systems [12]), system simulations are usually limited to the uncoded bit error rate (BER), e.g., [13], where the channel coding is omitted. A simple way to include the Turbo code in a LTE system (or any other powerful channel code) without increasing simulation complexity is to use the threshold receiver model: the block error rate (BLER) of a single-input single-output (SISO) system with Turbo code in an additive white Gaussian noise (AWGN) channel can be model as [9]

$$
P_{e}(\gamma)= \begin{cases}1, & \gamma<\gamma_{t h} \\ 0, & \gamma>\gamma_{t h}\end{cases}
$$

where $\gamma$ is the received signal to noise ratio (SNR) in the AWGN channel and $\gamma_{t h}$ is the threshold value. In a fading channel, the average BLER of the threshold receiver (1) is

$$
\bar{P}_{e}(\bar{\gamma})=\int_{0}^{\infty} P_{e}(\gamma) f(\gamma ; \bar{\gamma}) d \gamma
$$

where $\bar{\gamma}$ represents the average $\gamma$ and $f$ denotes the probability density function (PDF) of $\gamma$ for a given $\bar{\gamma}$. Substituting (1) into (2), one obtains

$$
\bar{P}_{e}(\bar{\gamma})=\int_{0}^{\gamma_{t h}} f(\gamma ; \bar{\gamma}) d \gamma=F\left(\gamma_{t h} ; \bar{\gamma}\right)
$$

where $F$ denotes the cumulative distribution function (CDF) of $\gamma$ for a given $\bar{\gamma}$. Interestingly, (3) can be justified by the outage theorems [11], where it is shown that, with powerful channel coding, the average BLER in a fading channel can be well approximated by the outage probability of the fading channel. For instance, in a Rayleigh fading environment (e.g., a well-stirred RC [14]), (3) becomes

$$
\bar{P}_{e}(\bar{\gamma})=1-\exp \left(-\frac{\gamma_{t h}}{\bar{\gamma}}\right) .
$$

As a result, we use CDF and BLER interchangeably in this paper.

The throughput of a SISO system with the hybrid automatic repeat request (H-ARQ) and fixed modulation and coding scheme (MCS) can be easily modeled as

$$
T_{\text {put }}(\bar{\gamma})=T_{\text {put }, \max }\left(1-\bar{P}_{e}(\bar{\gamma})\right)
$$

where $T_{\text {put,max }}$ denotes the maximum throughput. The maximum throughput with a fixed MCS is achieved at high SNR.

Since the OFDM effectively partitions the system bandwidth into subcarriers each experiencing a flat fading, throughout this paper, we express all channel models as if they were flat fading channels. In a frequency-selective fading channel, the corresponding channel model is regarded as that of a single OFDM subcarrier. For the OFDM with coding and interleaving across subcarriers, the SNR in the frequency-selective fading channel can be modeled by coherently combining the SNRs of independent OFDM subcarriers [7], assuming that the OFDM cyclic prefix is longer than the maximum delay in the fading channel. The number of independent subcarriers can be approximately estimated as the ratio of the system bandwidth to the coherence bandwidth of the channel. The cyclic delay diversity [12] can be approximated by coherently combing the spatial streams. The SISO throughput model (5), which is valid for both flat and frequency-selective fading channels, is the building block for developing throughput models of SIMO, MISO, and MIMO systems.

\section{B. Throughput Model for Diversity}

The throughput models for both SIMO and MISO systems can be readily obtained by determining the (empirical) CDF of the instantaneous SNR $\gamma$. So it suffices to model $\gamma$ in this subsection.

In an LTE system, the receiver is assumed to have the full channel state information (CSI) via channel estimation, whereas the transmitter does not have the full CSI due to the limited feedback in a frequency-division duplex (FDD) system. Thus, for a SIMO system, ${ }^{4}$ a maximum ratio combining (MRC) receiver is assumed [7]. The corresponding (instantaneous) SNR can be modeled as

$$
\gamma=\bar{\gamma}_{0} \mathbf{h}^{H} \mathbf{h}=\bar{\gamma}_{0}\|\mathbf{h}\|_{2}^{2}
$$

where $\bar{\gamma}_{0}=E\left[|x|^{2}\right] / E\left[\left|n_{i}\right|^{2}\right]$.

${ }^{4} \mathbf{y}=\mathbf{h} x+\mathbf{n}$, where $x, \mathbf{y}, \mathbf{h}$, and $\mathbf{n}$ are the transmit signal, receive signal vector, SIMO channel, and noise vector, respectively. Note that we assume independent identically distributed (i.i.d.) Gaussian noise throughout this paper. 
In a MISO LTE system, the space-frequency block code (SFBC) is used to enable transmit diversity in the absence of CSI. For a $1 \times 2$ LTE system, 5 the transmitter uses the Alamouti-like SFBC code [12],

$$
\left[\begin{array}{cc}
x_{k} & x_{k+1} \\
-x_{k+1}^{*} & x_{k}^{*}
\end{array}\right]
$$

where $x_{k}$ represents the $k$ th OFDM subcarrier, and the first and second rows correspond to the first and second transmit antennas, respectively. So the SNR of the $1 \times 2$ LTE system can be modeled as [15]

$$
\gamma=\frac{\bar{\gamma}_{0}\|\mathbf{h}\|_{2}^{2}}{2}
$$

Comparing (6) and (8), it is found that the SNR of the $1 \times 2$ LTE system is $3 \mathrm{~dB}$ lower than that of the $2 \times 1$ LTE system. This is because that the $2 \times 1$ SIMO has an array factor of 2 for knowing the CSI at the receive side [15].

\section{Throughput Model for Spatial Multiplexing}

Perhaps, the most important feature of an LTE system is the spatial multiplexing. Thus, a special focus of this work is put on modeling the spatial multiplexing throughput.

The MIMO channel (for a single OFDM subcarrier) can be modeled as

$$
\mathbf{y}=\mathbf{H} \mathbf{x}+\mathbf{n}
$$

where $\mathbf{H}$ is the MIMO channel matrix, $\mathbf{x}$ and $\mathbf{y}$ are the transmitted and received signal vectors, respectively, and $\mathbf{n}$ is the noise vector with i.i.d. Gaussian elements. It has been shown in [16] that the Kronecker model is as accurate as the full-correlation model [15] in an RC due to its rich scattering property. ${ }^{6}$ Therefore, the Kronecker model is used in this work:

$$
\mathbf{H}=\mathbf{R}_{r}^{1 / 2} \mathbf{H}_{w} \mathbf{R}_{t}^{1 / 2}
$$

where the $N_{R} \times N_{T}$ matrix $\mathbf{H}_{w}$ denotes the spatially white MIMO channel with i.i.d. complex Gaussian elements. The $N_{T} \times N_{T}$ matrix $\mathbf{R}_{t}$ and the $N_{R} \times N_{R}$ matrix $\mathbf{R}_{r}$ are the correlation matrices at the transmit and receive sides, respectively [18].

$$
\mathbf{R}_{r}=\boldsymbol{\Xi}_{r} \circ \boldsymbol{\Phi}_{r}
$$

where the $N_{R} \times N_{R}$ matrix $\boldsymbol{\Xi}_{r}=\sqrt{\mathbf{e}_{r}}{\sqrt{\mathbf{e}_{r}}}^{T}$ with $\mathbf{e}_{r}$ denoting a column vector consisting the antenna efficiencies at the receive side, $\sqrt{ }$ represents element-wise square root, $\circ$ denotes element-wise product, and the antenna correlation matrix $\boldsymbol{\Phi}_{r}$ consists of the complex antenna correlation coefficients between the receive antennas. $\mathbf{R}_{t}$ can be determined similarly. Note that the antenna correlation is the correlation of the composite channel including both spatial correlation and antenna mutual coupling effects. In this work, all correlation is referred to as antenna correlation in order to distinguish it from the spatial correlation of

\footnotetext{
${ }^{5}$ In this paper, a MIMO system is expressed as $N_{R} \times N_{T}$, where $N_{R}$ and $N_{T}$ are the number of receive and transmit antennas, respectively.

${ }^{6}$ In real-life environments, the Kronecker model may not be as accurate as the full-correlation model or the Weichselberger models [17] in that it neglects the joint spatial structure of the MIMO channel.
}

the spatial channels without the antenna effects. Also note that in an isotropic-scattering environment such as the RC, the spatial correlation is a Sinc function of the distance between the observation points, e.g., [21]. We use good, nominal, and bad CTIA reference antennas in the measurements in order to have a predefined antenna correlation. In a general fading environment, $\boldsymbol{\Phi}_{r}$ can be determined by taking into account of both the angular distribution and the cross polarization ratio, see, e.g., (21) in [18]. Nevertheless, the model is confined to the slow fading case.

Depending on the availability of the partial CSI at the transmitter, MIMO systems can be categorized as open- and closedloop configurations. We discuss these two configurations separately in the sequel.

1) Open-Loop Configuration: In an open-loop MIMO system with full spatial multiplexing (i.e., the number of independent data streams equals the number of transmit antennas $N_{T}$ ), the transmitter does not have CSI. Thus, there is no precoding. Let $\mathbf{h}_{i}$ be the $i$ th column of $\mathbf{H}$, (9) can be rewritten as

$$
\mathbf{y}=\mathbf{h}_{i} x_{i}+\sum_{j \neq i} \mathbf{h}_{j} x_{j}+\mathbf{n} .
$$

The first term in the right side of (12) stands for the received signal of the $i$ th stream, and the second term represents the interferences from all the other streams with respect to (w.r.t.) the $i$ th stream. We use ZF, MMSE, and ML receivers, respectively, to cope with interferences and noises.

$Z F$ Receiver: The $Z F$ receiver for the $i$ th stream corresponds to the $i$ th row of the pseudo-inverse of $\mathbf{H}$, $\left(\mathbf{H}^{H} \mathbf{H}\right)^{-1} \mathbf{H}^{H}$. It projects the $i$ th data stream into the subspace orthogonal to the one spanned by columns $\mathbf{h}_{j}\left(j=1, \ldots, N_{T}\right.$ and $j \neq i$ ). Assuming equal power allocation and left multiplying the pseudo-inverse of $\mathbf{H}$ to both sides of (9), the ZF output SNR of the $i$ th stream can be easily derived as

$$
\gamma_{i}=\frac{\bar{\gamma}_{0}}{\left[\left(\mathbf{H}^{H} \mathbf{H}\right)^{-1}\right]_{i, i}}
$$

where $\bar{\gamma}_{0}=E\left[\left|x_{i}\right|^{2}\right] / E\left[\left|n_{i}\right|^{2}\right]$. The MIMO throughput is the sum of the throughputs of all the streams:

$$
T_{\text {put }}(\bar{\gamma})=\sum_{i} T_{\text {put }, \text { max }, i}\left(1-\bar{P}_{e}\left(\bar{\gamma}_{i}\right)\right)
$$

where $T_{\text {put,max }, i}=T_{\text {put,max }} / N_{T}$ and $\bar{\gamma}_{i}=\bar{\gamma} / N_{T}$.

MMSE Receiver: The ZF receiver completely eliminates the interference at the expense of noise enhancement. The MMSE receiver improves the SNR performance by making a tradeoff between interference and noise mitigations. Instead of using the pseudo-inverse of $\mathbf{H}$ as the filtering matrix, the MMSE receiver finds a filtering matrix $\mathbf{W}$ that minimizes $|\mathbf{W y}-\mathbf{x}|_{2}^{2}$. W can be easily derived using the orthogonality principle [15]. Left multiplying $\mathbf{W}$ to both sides of (9), the MMSE (output) SNR can be readily derived as

$$
\gamma_{i}=\frac{1}{\left[\left(\bar{\gamma}_{0} \mathbf{H}^{H} \mathbf{H}+\mathbf{I}\right)^{-1}\right]_{i, i}}-1 .
$$


Note that in this paper we will not distinguish SNR and signal to interference and noise ratio (SINR) in that the inter-stream interference can be regarded as noise.

The throughput model for the MMSE receiver can be obtained by replacing the ZF SNR in (14) with the MMSE SNR.

ML Receiver: Both ZF and MMSE are suboptimal receivers, but they enjoy low decoding-complexity in that they are both linear receivers. The ML receiver is optimal, but its decoding complexity can be prohibitive. The sphere decoding approximately achieves the ML performance yet with much lower complexity. However, its complexity is still much higher than the linear receivers. A closed-form expression of the ML (or sphere decoding) SNR does not exist. However, according to the IEEE 802.16e standard [19], the ML SNR can be estimated as

$$
\gamma=\exp \left\{\frac{1}{N_{T}} \ln \left[\operatorname{det}\left(\mathbf{I}+\bar{\gamma}_{0} \mathbf{H}^{H} \mathbf{H}\right)\right]\right\}-1
$$

where $\ln$ denotes the natural logarithm (i.e., $\log _{e}$ ). Obviously, (16) results from a heuristic approach, presuming that an ML receiver achieve the capacity. Note that the relevance of the SNR estimator (16) can be partially justified by taking the example of a SIMO system: it is easy to show that the MRC SNR (6) can be obtained by using (16).

The throughput model for the ML receiver can be obtained by replacing the SNR in (5) with the ML SNR. The throughput performances of the three receivers are compared in Section III.

2) Closed-Loop Configuration: In a closed-loop MIMO (LTE) system with FDD, there is limited feedback (i.e., only partial CSI is available at the transmitter). The transmitter uses the codebook-based precoding [12], $\mathbf{x}=\mathbf{F s}$, where $\mathbf{s}$ is the transmit signal vector before precoding and $\mathbf{F}$ is the precoding matrix. For a $2 \times 2$ LTE system, the receiver feedbacks two bits to the transmitter. Based on the two bits, the transmitter chooses a precoding matrix $\mathbf{F}$ from the following codebook [12]:

$$
\left[\begin{array}{ll}
1 & 0 \\
0 & 1
\end{array}\right], \quad \frac{1}{\sqrt{2}}\left[\begin{array}{cc}
1 & 1 \\
1 & -1
\end{array}\right], \quad \frac{1}{\sqrt{2}}\left[\begin{array}{cc}
1 & 1 \\
j & -j
\end{array}\right] .
$$

The selection criterion of the precoding matrix $\mathbf{F}$ can be based on the trace of the mean square error (MSE) matrix (18) or the capacity (19) [15].

$$
\begin{aligned}
M S E(\mathbf{F}, \mathbf{W}) & =E\left[(\mathbf{W} \mathbf{y}-\mathbf{s})(\mathbf{W} \mathbf{y}-\mathbf{s})^{H}\right] . \\
C(\mathbf{F}) & =\log _{2}\left[\operatorname{det}\left(\mathbf{I}+\bar{\gamma}_{0} \mathbf{F}^{H} \mathbf{H}^{H} \mathbf{H F}\right)\right] .
\end{aligned}
$$

Note that $\mathbf{F}$ is implicitly included in $\mathbf{y}$ in (18). For an MMSE receiver, (18) reduces to

$$
M S E(\mathbf{F})=\bar{\gamma}_{0}\left(\mathbf{I}+\bar{\gamma}_{0} \mathbf{F}^{H} \mathbf{H}^{H} \mathbf{H F}\right)^{-1} .
$$

After choosing the precoding matrix $\mathbf{F}$, the SNRs of ZF, MMSE, and ML receivers can be calculated by replacing $\mathbf{H}$ in (13), (15), and (16) with the effective channel HF, respectively.

Note that, for the sake of easy exhibition, we use the relative throughput, defined as the throughput normalized by its maximum value, $T=T_{\text {put }} / T_{\text {put,max }}$, hereafter.

\section{Spatial Multiplexing Efficiency}

The throughput models developed in Section II implicitly include the antenna effects (such as antenna correlations and efficiencies) via (10) and (11). ${ }^{7}$ In order to conveniently characterize antenna effects on the throughput, we resort to the spatial multiplexing (SM) efficiency [10], ${ }^{8}$ defined as the SNR degradation, in the presence of imperfect antennas w.r.t. that of an ideal fading channel (i.e., i.i.d. channel with ideal antennas) at $95 \%$ throughput level. Mathematically, it can be expressed as

$$
e_{\text {mux }}=\left.\frac{T_{0}^{-1}(\bar{\gamma})}{T^{-1}(\bar{\gamma})}\right|_{95 \%}
$$

where $T^{-1}$ denotes functional inversion of the relative throughput $T$, and $T_{0}$ represents the relative throughput for the i.i.d. fading channel with ideal antennas. The choice of the $95 \%$ throughput level is motivated by the fact that $95 \%$ throughput is usually of interest [1]. Using the developed throughput models, the SM efficiencies for different receivers are shown in Section III.

\section{SimUlations}

In order to characterize the device under test (DUT) alone as a receiver in the downlink mode, the antennas of the base station (transmitter) are assumed to be uncorrelated. Therefore, we mainly focus on the receive semi-correlation case, i.e., without further specifications, receive semi-correlation is assumed. Note that the semi-correlation is related to the Kronecker model (cf. Section II-C). In reality, when the Kronecker model assumption does not hold, the semi-correlation may not correspond to the true correlation of the MIMO channel. Nevertheless, as mentioned before, due to the rich isotropic scattering property of the $\mathrm{RC}$, the semi-correlation is the antenna correlation measured at either MIMO end in the RC. For simplicity, we assume flat fading channels in this section. Frequency-selective channels are considered in the next section for modeling RC measurements.

For simulations, we first generate i.i.d. Gaussian matrix $\mathbf{H}_{w}$ with 10000 snapshots and introduce power imbalance and antenna correlation to the MIMO channel via (10) and (11). Based on the channel snapshots, 10000 instantaneous SNR samples are gathered for ZF, MMSE, and ML receivers in open- and closed-loop configurations, respectively; and the corresponding empirical CDF (or the average BLER) is obtained using all the 10000 SNR samples. Once the average BLER is obtained, the throughput can be calculated easily using the corresponding throughput model.

First we use a $2 \times 2$ open-loop MIMO system to compare the throughput performances of ZF, MMSE, and ML receivers. Fig. 1 shows the relative throughput of each receiver with ideal and imperfect $(0.7$ antenna correlation and $6-\mathrm{dB}$ power imbalance) antennas, respectively. As can be seen, compared with the optimal ML receiver, the linear ZF and MMSE receivers incur

\footnotetext{
${ }^{7}$ The antenna correlations and efficiencies are included in the correlation matrix (11), which is in turn included in the Kronecker channel model (10).

${ }^{8}$ The SM efficiency was first originally proposed in [10] w.r.t. MIMO capacity instead of throughput.
} 


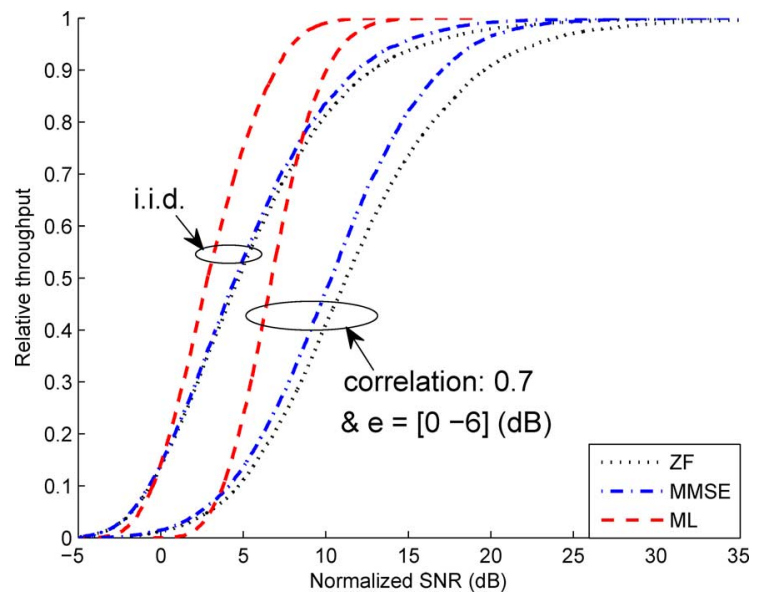

Fig. 1. Relative throughput of $2 \times 2$ open-loop MIMO systems with ZF, MMSE, and ML receivers. The normalized SNR is defined $\bar{\gamma} /\left(\gamma_{t h} \bar{\gamma}_{0}\right)$, where $\bar{\gamma}_{0}=15 \mathrm{~dB}$ is assumed.

losses in both SNR and spatial diversity. The former is manifested as a shift of the throughput curve to the right; the latter can be seen from the slope of the throughput curve at high SNR. Note that the diversity orders of ZF and MMSE receivers are (approximately) $N_{R}-N_{T}+1$, where as the spatial diversity order of the ML receiver equals the number of receive antennas $N_{R}$ (independent of the number of transmit antennas $N_{T}$ ) [15]. Also note that the throughputs of the MMSE and ML receivers depends on the parameter $\bar{\gamma}_{0}=E\left[\left|x_{i}\right|^{2}\right] / E\left[\left|n_{i}\right|^{2}\right]$, whereas the $\mathrm{ZF}$ throughput is independent of $\bar{\gamma}_{0}$. This can be explained by looking at the SNR expressions of the three receivers, i.e., (13), (15), and (16).

To show the antenna effects on the throughput, we plot the SM efficiency as a function of the antenna correlation and the power imbalance for the three receivers in Fig. 2, respectively. In order to illustrate the throughput dependences of the MMSE and ML receivers on $\bar{\gamma}_{0}$, the SM efficiencies for 15- and 25-dB $\bar{\gamma}_{0}$ are shown in Fig. 2 for the two receivers. As can be seen, the ZF SM efficiency expressions (i.e., $1-|\rho|^{2}$ and $2\left(1 / e_{1}+\right.$ $\left.1 / e_{2}\right)^{-1}$ [8]) well agree with the simulated ones. The cyan asterisks in the left graph represent the SM efficiency of an LTE device measured in an RC (cf. Section V). SM efficiencies with $\mathrm{ZF}$ and MMSE (with $25-\mathrm{dB} \bar{\gamma}_{0}$ ) receivers agree with that of the $\mathrm{RC}$ measurement, while the ML receiver clearly underestimate the power imbalance effect on the throughput. This is because the LTE terminals are equipped with linear equalizers in order to avoid the high complexity of the ML decoder [12].

We compare the throughputs of the open- and closed-loop MIMO systems in Fig. 3. For the sake of conciseness of the paper, we confine to the throughput of the MMSE receiver (similar results are obtained for the ZF receiver). Following the LTE standard [12], the transmitter in the closed-loop configuration chooses a precoding matrix from (17) based on 2-bit feedback. Fig. 3 shows the throughputs of the open- and closed-loop configurations for the transmit semi-correlation case, where the trace of the MSE (lower graph) and the capacity (upper graph) are used as the criteria for selecting the precoding matrix. As can be seen, the precoding improves the throughput at low SNR (at high SNR, there is no improvement by using

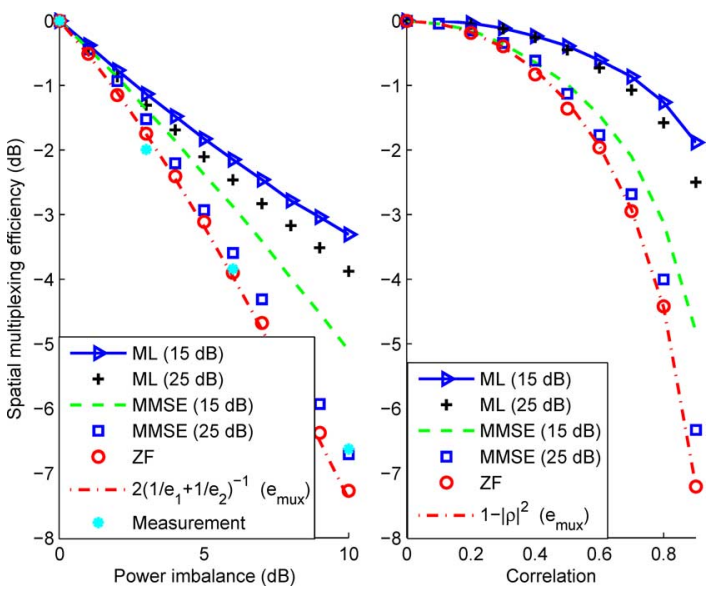

Fig. 2. SM efficiencies (of a $2 \times 2$ open-loop MIMO system with ZF, MMSE, and ML receivers) as functions of power-imbalance and correlation. The $\mathrm{dB}$ values in the brackets after ML and MMSE represent the value of $\bar{\gamma}_{0}$. The cyan asterisks denote the measured SM efficiency of DUT A in an RC (cf. Section V).
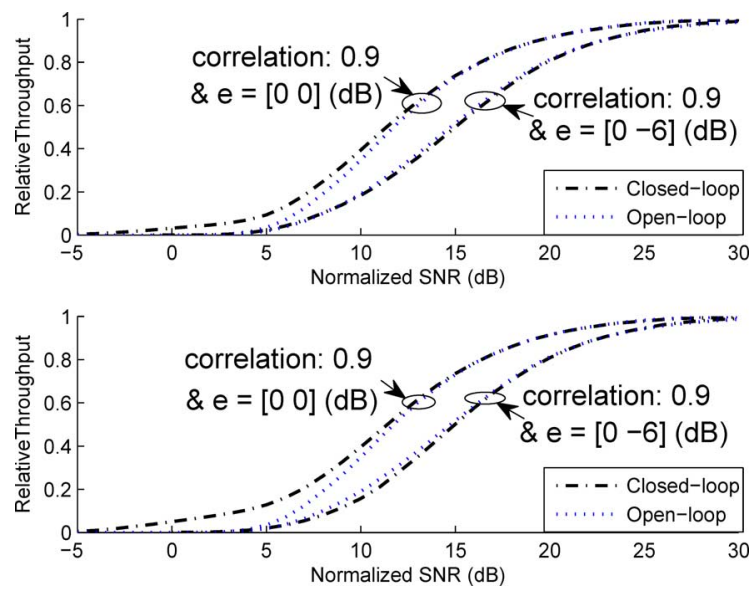

Fig. 3. Relative throughputs of $2 \times 2$ open- and closed-loop (with 2-bit feedback) MIMO systems for the transmit semi-correlation case. The upper graph corresponds to the capacity precoding selection criterion (19); the lower graph corresponds to the trace of the MSE precoding selection criterion (20).

precoding, since it is optimal to transmit independent streams from the transmit antennas at high SNR [15]) and that the two criteria (19) and (20) yield similar results. Hence, we will use the MSE criteria (20) for modeling closed-loop throughput in the next section.

\section{MEASUREMENTS}

To validate the developed throughput models in Section II, SIMO, MISO, and MIMO LTE systems are measured in an RC. The $2 \times 2$ MIMO throughput measurement setup is shown in Fig. 4 (SIMO and MISO throughput measurement setups are similar except that only one antenna is used at transmit and receive sides, respectively.) A commercial communication tester is used as the LTE base station. The DUTs are a commercial LTE USB modem and an LTE mobile phone (referred to as DUT A and DUT B hereafter, respectively) with external antenna ports for OTA testing.

DUT A was located in a separate shielded box outside the $\mathrm{RC}$ during the measurements. It was connected with two discone antennas (via two RF cables) that are located inside the 


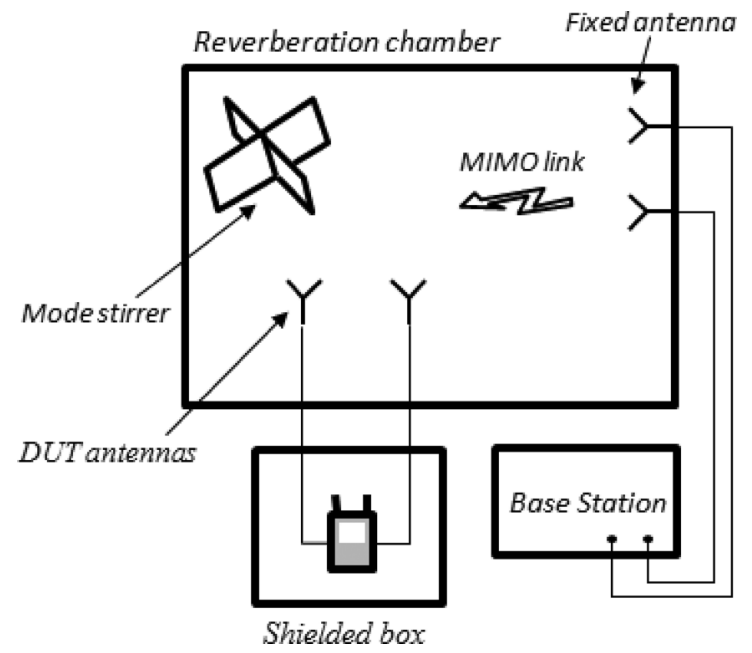

Fig. 4. Measurement setup for LTE MIMO throughput tests.

RC. The two discone antennas were kept uncorrelated by putting them orthogonal to each other and with sufficient separation. Only power imbalance was introduced at the receive side by connecting a 3-, 6-, or $10-\mathrm{dB}$ attenuator to one of the discone antenna for each throughput measurement of DUT A. This setting allows us to examine the power imbalance effect on the throughput solely. Measurements of DUT A were performed on the LTE band 7, i.e., $2655 \mathrm{MHz}$, with 10-MHz system bandwidth in open-loop configuration. The LTE system was set to a fixed 64 QAM modulation with maximum rates of about 20 and $40 \mathrm{Mbps}$ for the diversity (SIMO and MISO) measurements and the spatial multiplexing measurements, respectively.

In order to include the antenna correlation effect in the throughput measurements as well, DUT B was connected with the two-port power-balanced good, nominal, and bad CTIA reference antennas (e.g., [22]) that were located inside the RC. Note that the CTIA reference antennas [22] include RF shielding boxes in which DUT B was placed. Measurements of DUT B were performed on the LTE band 13, i.e., $751 \mathrm{MHz}$, with $10-\mathrm{MHz}$ system bandwidth in closed-loop configuration. The LTE system was set to a fixed 64 QAM modulation with maximum rates of about $35 \mathrm{Mbps}$ for the spatial multiplexing measurements. The magnitudes of the complex (antenna) correlation coefficients of the good, nominal, and bad CTIA reference antennas are about $0.13,0.57$, and 0.90 , respectively. The antenna efficiencies of the good, nominal, and bad CTIA reference antennas are about $-1.4,-3.1$, and $-4.0 \mathrm{~dB}$, respectively.

The measurements of both DUTs were conducted for the downlink communications. The two fixed transmit antennas (or OTA antennas [1]) in the RC are power-balanced and uncorrelated. Note that the transmit antenna efficiencies and the pass loss are calibrated out by performing a reference measurement a priori.

Furthermore, in order to study the antenna impairments at the transmit side another measurement was performed, where the DUT B was connected to the good CTIA reference antenna and the base station was connected to the bad CTIA reference antenna. Other measurement settings are the same as the previous measurements of the DUT B.
It is well-known that the coherence bandwidth affects the number of independent OFDM subcarrier channels (and therefore the frequency diversity of the OFDM system). A smaller coherence bandwidth results in a larger slope of the measured throughput (up to a limit where the maximum time delay equals the length of the cyclic prefix of the OFDM symbols [15]). During the measurement, the $\mathrm{RC}$ was loaded to achieve an RMS delay spread of $90 \mathrm{~ns}$ (corresponding to 3-MHz coherence bandwidth) [23], [24]. This specific loading was chosen because it is close to that of a typical indoor environment [2], [25]. The OFDM effectively partitions the wideband channel into subcarrier channels each experiencing a Rayleigh-flat fading in the $\mathrm{RC}$ [14]. Intuitively the number of independent subcarrier channels can be approximated by dividing the system bandwidth (10 MHz) by the coherence bandwidth $(3 \mathrm{MHz})$, resulting in a 3-order frequency diversity. However, it was found experimentally that the 2-order frequency diversity gives better agreement with the measurement of a $2 \times 1$ LTE system than that of the 3-order frequency diversity for the same RC loading [7]. As a result, the 2-order frequency diversity was assumed in modeling the $2 \times 2$ spatial multiplexing throughput for the same loading [8] without further investigation. By comparing the spatial multiplexing throughput shown later in this section (which assumes a 3-order frequency diversity) with that shown in [8], it can be seen that the 3-order frequency diversity model actually gives better agreement with the measured spatial multiplexing throughput. The reasons are that the MRC diversity of the $2 \times 1$ SIMO system tends to increase the post-processing coherence bandwidth (i.e., the channel seen at the MRC output has a larger coherence bandwidth than that seen at a single receive antenna port) and that a $2 \times 2$ MIMO system with full spatial multiplexing has no extra degree-of-freedom for the spatial diversity. This phenomenon can be explained intuitively: the MRC diversity (or receive beamforming) tends to steer the beam to the dominant path and spatially filters secondary paths from other directions arriving at later times, which effectively reduces the delay spread (or equivalently increases the coherence bandwidth) at the MRC output. Experimental verification of this is shown in the Appendix.

Note that only absolute power values are available from the OTA testing instrument. Thus, the measured throughputs are shown as a function of the received (average) power $\bar{P}$. In simulations, the relative throughput is presented as a function of the normalized SNR. To compare the modeled relative throughput with the measured one, we need to correct the $\mathrm{x}$-axis of the simulated curve with the absolute threshold value $P_{t h}, P_{t h} \bar{\gamma}_{0} / \bar{\gamma}$. The threshold value $P_{t h}$ was obtained from the conductive measurement (i.e., the transmitter and the receiver are connected via RF cables). The conductive measurements have been shown in [7], [8]. The threshold values $(-83.7$ and $-78.9 \mathrm{dBm}$ for DUT A and DUT B, respectively) are read out from the $50 \%$ of the maximum conductive throughput. For the sake of conciseness, the conductive measurement results are not shown in this paper. Note that almost all the baseband algorithms are calibrated out by performing a conductive measurement of the same DUT (according to the threshold receiver model, see Section II) except for the spatial equalizer. Because the conductive measurement creates two interference-free (parallel) channels by connecting 


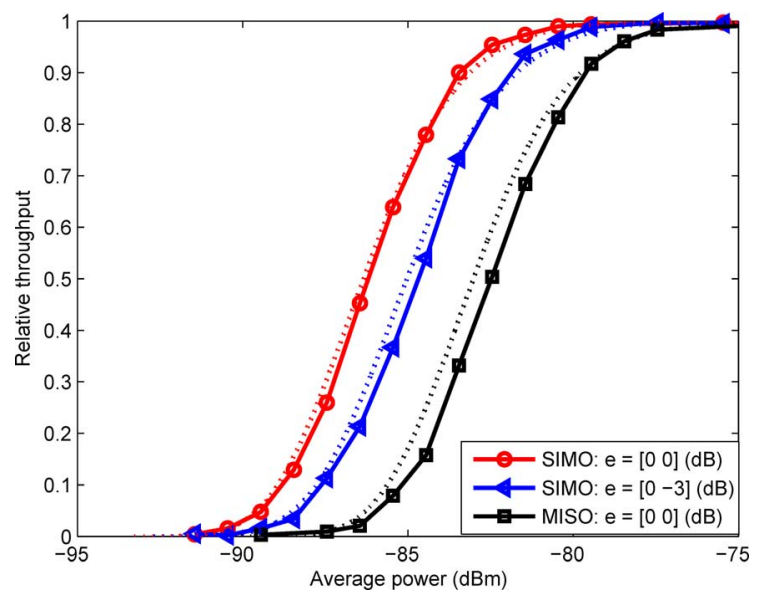

Fig. 5. Relative throughput of $2 \times 1$ SIMO and $1 \times 2$ (open-loop) MISO systems with DUT A in the RC. Solid curves represent measurements; dotted curves represent the corresponding throughput models.

two RF cables between the communication tester and the DUT, it does not take into account of the inter-stream interference. Thus, the spatial equalizer (e.g., ZF, MMSE, or ML) has to be included in modeling the throughput in the fading channel. Also note that it is our intention to exclude most of the baseband algorithms (by approximating them using the threshold receiver) in throughput modeling, which greatly simplifies the throughput simulation and allows us to focus on the antenna effects on the LTE throughput in a fading channel.

Fig. 5 shows the measured and modeled relative throughputs for the SIMO and MISO cases, where e $=[0-3] \mathrm{dB}$ represents the case where a 3-dB attenuator is connected to one of the receive antennas. There are good agreements between the measured and modeled throughputs. Note that the power-balanced MISO throughput curve shifts about $3 \mathrm{~dB}$ to the right, compared with the power-balanced SIMO throughput. This is due to the fact that the $2 \times 1$ SIMO system has a 3 -dB array factor (in that the CSI is known at the receiver), whereas the $1 \times 2$ MISO system does not (for the transmitter does not know the CSI). Note that the SFBC is used to achieve the transmit diversity for the MISO case in the absence of CSI at the transmitter (cf. Section II-B).

The measured SM efficiency of the $2 \times 2$ open-loop MIMO system with DUT A has been shown in Fig. 2. As can be seen, the modeled SM efficiencies with the ZF and MMSE (with $25-\mathrm{dB} \bar{\gamma}_{0}$ ) receivers well agree with the measured one, and that the ML underestimates the power imbalance effect. Note that, although adding the 2-order frequency diversity increases the slope of the throughput curve, it has little effect on the SM efficiency since the SM efficiency is defined as the relative SNR degradation due to the imperfect MIMO antenna w.r.t. the ideal i.i.d. case. As a result, we only model the MIMO throughput using the $\mathrm{ZF}$ and MMSE receivers in this section. The throughput model with the ML receiver not only predicts a larger spatial diversity order but also results in a sever mismatch for the power-unbalanced case.

Fig. 6 shows the measured and modeled relative throughputs for the $2 \times 2$ open-loop MIMO system with DUT A (connected to two external discone antennas with one of them cascaded with
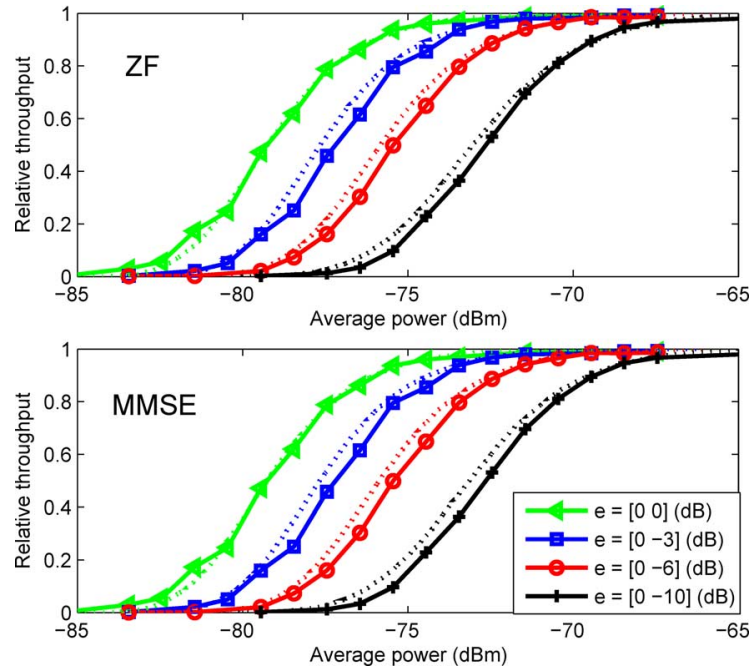

Fig. 6. Relative throughputs of $2 \times 2$ open-loop MIMO system with DUT A. Solid curves represent measurements; dotted curves represent the throughput models with ZF receiver (upper) and MMSE receiver with $25-\mathrm{dB} \bar{\gamma}_{0}$ (lower)

a 0-, 3-, 6-, and 10-dB attenuator, respectively). As can be seen, the MIMO throughput models with the ZF and MMSE receivers reasonably predict the measured throughput. Note that the assumption of $25-\mathrm{dB} \bar{\gamma}_{0}$ (i.e., $E\left[\left|x_{i}\right|^{2}\right] / E\left[\left|n_{i}\right|^{2}\right]$ ) for the MMSE case can be justified by the fact that a high transmit power is required to support the dense (high rate) 64 QAM modulation constellation. Note that $\bar{\gamma}_{0}$ in (15) is the SNR without the antenna impairment. So the actual SNR at the MMSE receiver output is smaller than $\bar{\gamma}_{0}$ due to the adverse effect of the antennas. It is found experimentally (by sweeping $\bar{\gamma}_{0}$ ) that once $\bar{\gamma}_{0}$ is larger than $25 \mathrm{~dB}$, the MMSE receiver results in good agreement with the measurement. For an even larger $\bar{\gamma}_{0}$ (e.g., $30 \mathrm{~dB}$ ), the result is almost the same. One can use the same method to experimentally determine the appropriate $\bar{\gamma}_{0}$ for another modulation. By performing extra throughput measurements with lower modulation orders, we found that $20-\mathrm{dB} \bar{\gamma}_{0}$ is enough to model 16QAM, and $15-\mathrm{dB} \bar{\gamma}_{0}$ for QPSK.

Now that these empirical values are found, one can use them directly for the MMSE-based throughput modeling later. Also note that one can use the $\mathrm{ZF}$ receiver to avoid the need of knowing $\bar{\gamma}_{0}$ in the throughput modeling. It should also be noted that, for DUT A, the maximum throughput of the MIMO multiplexing case is $40 \mathrm{Mbps}$, which is twice of that of the SIMO or MISO case. Bearing this in mind, comparing the $\mathrm{x}$-axes of Figs. 5 and 6 for the power-balanced case, it can be seen that more power is needed in order to achieve the spatial multiplexing and that, with insufficient received power (e.g., between -85 and $-80 \mathrm{dBm}$ ), the diversity scheme offers higher (absolute) throughput than the spatial multiplexing.

Fig. 7 shows the measured and modeled relative throughputs for the $2 \times 2$ closed-loop MIMO system with DUT B (connected to the external two-port good, nominal, and bad CTIA reference antennas, respectively). As expected, good agreements are observed for all the three CTIA reference antennas.

Fig. 8 shows the measured and modeled relative throughputs for the $2 \times 2$ open-loop MIMO system with DUT B connected to the good CTIA reference antenna and the base station connected 

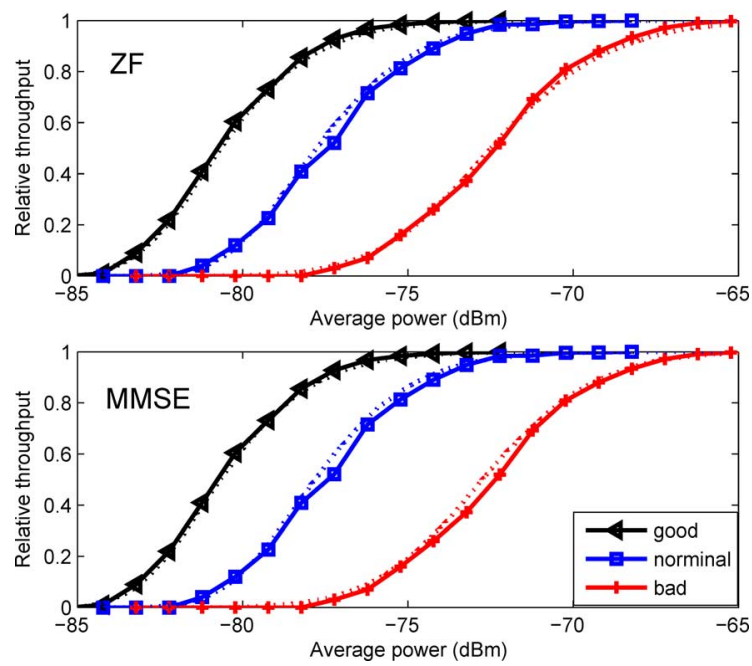

Fig. 7. Relative throughputs of $2 \times 2$ closed-loop MIMO system with DUT B. Good, nominal, and bad denotes the good, nominal, and bad CTIA reference antennas, respectively. Solid curves represent measurements; dotted curves represent the throughput models with ZF receiver (upper) and MMSE receiver with 25 -dB $\bar{\gamma}_{0}$ (lower).
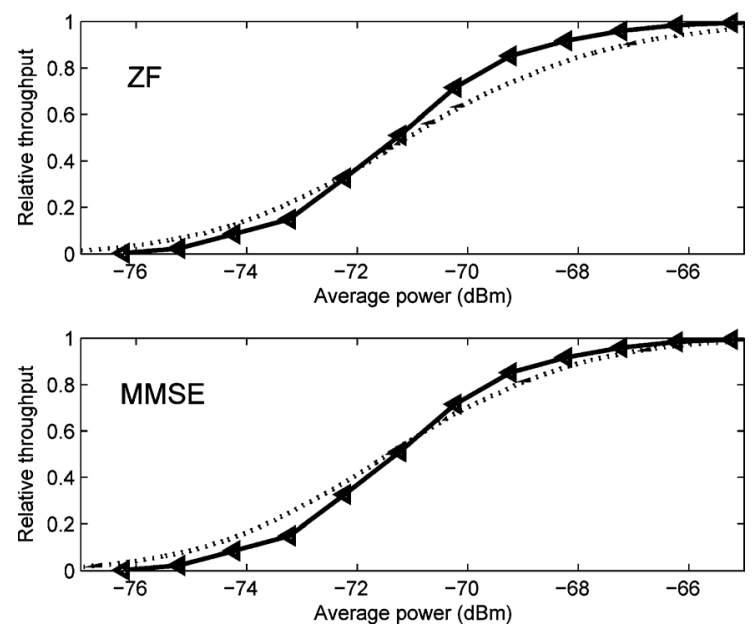

Fig. 8. Relative throughputs of $2 \times 2$ open-loop MIMO system with DUT B connected to the good CTIA reference antenna and the base station connected to the bad CTIA reference antenna. Solid curves represent measurements; dotted curves represent the throughput models with ZF receiver (upper) and MMSE receiver with 25 -dB $\bar{\gamma}_{0}$ (lower).

to the bad CTIA reference antenna. There is reasonable agreement between the measured and modeled throughputs. The measure throughput is similar to that of the bad CTIA reference antenna at the receive side in Fig. 7; the slight shift of the measured throughput in Fig. 8 (as compared with that in Fig. 7) is due to the antenna efficiency and the antenna correlation of the good CTIA reference antenna. The procedure for generating the simulated throughput of Figs. 7 and 8 is the same. We first generate 10000 correlated channel realizations including the characteristics of the CTIA reference antennas via (10) and (11). Then we calculate the instantaneous SNRs of ZF and MMSE receivers by (13) and (15), respectively, for each channel realization. We obtain the empirical CDFs (or the average BLER) from the 10000 instantaneous SNR samples for both ZF and MMSE receivers. The relative throughputs of the ZF and MMSE receivers are readily obtained once their empirical CDFs are determined.

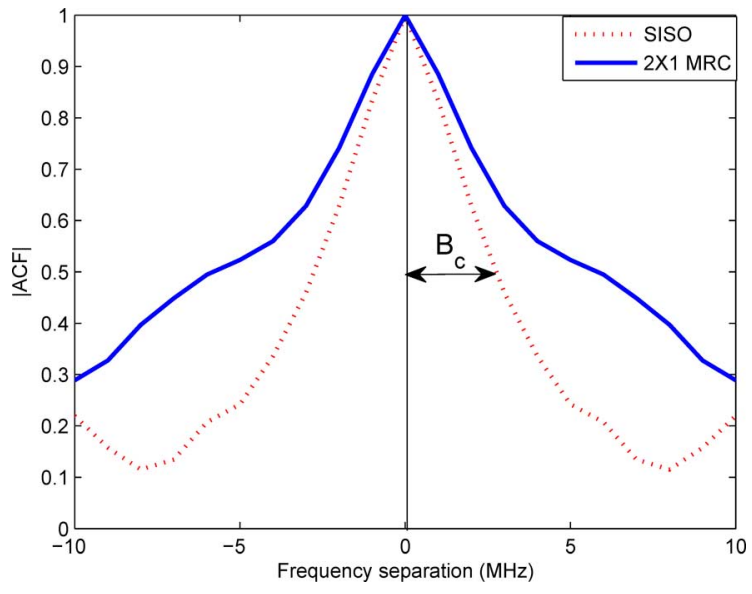

Fig. 9. ACF of a SISO channel and that seen at the output of a 2-port MRC antenna at LTE band 13. The coherence bandwidth (defined in [26]) of the SISO channel is marked in the figure.
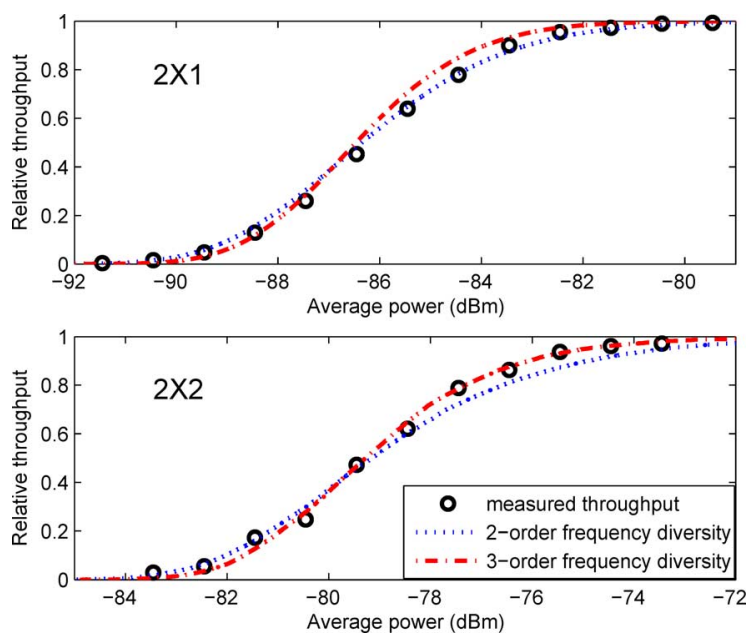

Fig. 10. Throughput models with 2-order and 3-order of frequency diversities for $2 \times 1$ diversity (upper) and $2 \times 2$ spatial multiplexing (lower), compared with measured throughputs of DUT A with two external discone antennas that are uncorrelated and power-balanced.

\section{CONCLUSION}

In this paper, we present simple throughput models with ZF, MMSE, and ML receivers, respectively. The throughput models get rid of the time-consuming simulation of the channel code (e.g., Turbo code) by using the empirical CDF of the equalized SNR, which is fast to obtain by simulations. The throughput model includes the antenna effects implicitly. In order to explicitly show the antenna effects on the throughput, we resort to the SM efficiency. In addition to open-loop configuration and receive semi-correlation, closed-loop configuration and transmit semi-correlation are also investigated. Measurement results show that the ML receiver underestimates the antenna impairment on the throughput and the throughput models with ZF and MMSE receivers well predict the measurements. Moreover, the antenna diversity effect on the coherence bandwidth (and therefore the system frequency diversity order) is studied, resulting in better agreements between the measured and modeled throughputs. The throughput models developed in this paper can be used to double check RC measurement results 
to eliminate potential systematic errors. They can also be used to complement RC measurements by predicting the throughput with arbitrary antenna characteristics and certain equalizers.

\section{APPENDIX \\ COHERENCE BANDWIDTH With/Without ANTENNA DIVERSITY}

In order to show the MRC diversity effect on the coherence bandwidth, we performed passive diversity measurement (from 650 to $2700 \mathrm{MHz}$ ) using two reference discone antennas as a two-port MRC diversity antenna with the same RC loading as that for the active throughput measurement. The channel transfer function can be readily obtained by sounding the channel with a vector network analyzer. The coherence bandwidth, $B_{c}$, can be determined from the autocorrelation function (ACF) of the channel transfer function [26]. Different $B_{c}$ definitions exist, this work use the definition given in [26]: $B_{c}$ is defined as the frequency separation where the magnitude of the ACF drops by $3 \mathrm{~dB}$, as shown in Fig. 9 for the SISO channel. Based on this definition, $\mathrm{B}_{\mathrm{c}}$ is related to the RMS delay spread $\sigma_{\tau}$ as $\mathrm{B}_{\mathrm{c}}=\sqrt{3} /\left(2 \pi \sigma_{\tau}\right)$ in the RC [23].

Fig. 9 shows the ACF of the channel transfer function seen by a single antenna and by a 2-port MRC diversity antenna at the center frequency of the LTE band 13, i.e., $751 \mathrm{MHz}$. (For the latter case, the MRC filter, $\mathbf{h}^{H} /\|\mathbf{h}\|_{2}$, is applied to the diversity channel $\mathbf{h}$ to obtain the channel seen at the MRC output.) Note that similar ACFs are observed at the center frequency of LTE band 7, i.e., $2655 \mathrm{MHz},{ }^{9}$ and these ACFs are not shown for the conciseness of the paper.

As can be seen, the coherence bandwidth seen at the MRC output is larger than that seen by a single antenna. Interestingly, this phenomenon has been observed in [27] for millimeter-wave beamforming. According to Fig. 9, the coherence bandwidth is about $3 \mathrm{MHz}$ for the SISO system (or systems without spatial diversity) and $6 \mathrm{MHz}$ at the output of a 2-port MRC diversity antenna. For an OFDM system with $10 \mathrm{MHz}$ bandwidth, the corresponding frequency diversity orders are $10 / 3 \approx 3$ and $10 / 6 \approx 2$, respectively. This finding is in agreement with the experimental observations that the 2-order frequency diversity in the throughput model gives the best agreement with the measured $2 \times 1$ diversity throughput and the 3 -order frequency diversity gives the best agreement with the measured $2 \times 2$ spatial multiplexing throughput (see Fig. 10). Note that there will be worse agreement if no frequency diversity is used in the model [7].

\section{REFERENCES}

[1] A. A. Glazunov, V. M. Kolmonen, and T. Laitinen, "MIMO overthe-air testing," in LTE-Advanced and Next Generation Wireless Networks: Channel Modelling and Propagation. Hoboken, NJ, USA: Wiley, 2012, pp. 411-441.

[2] E. Genender, C. L. Holloway, K. A. Remley, J. M. Ladbury, G. Koepke, and H. Garbe, "Simulating the multipath channel with a reverberation chamber: Application to bit error rate measurements," IEEE Trans. Electromagn. Compat., vol. 52, pp. 766-777, 2010.

\footnotetext{
${ }^{9}$ This is as expected in that the coherence bandwidth is almost frequency in-
} dependent from 600 to $3000 \mathrm{MHz}$ in the used RC, e.g., [23].
[3] G. Ferrara, M. Migliaccio, and A. Sorrentino, "Characterization of GSM non-line-of-sight propagation channels generated in a reverberating chamber by using bit error rates," IEEE Trans. Electromagn. Compat., vol. 49, no. 3, pp. 467-473, Aug. 2007.

[4] M. Á. García-Fernández, J. D. Sánchez-Heredia, A. M. MartínezGonzález, D. A. Sánchez-Hernández, and J. F. Valenzuela-Valdés, "Advances in mode-stirred reverberation chambers for wireless communication performance evaluation," IEEE Commun. Mag., vol. 49, no. 7, pp. 140-147, Jul. 2011.

[5] R. Recanatini, F. Moglie, and V. M. Primiani, "Performance and immunity evaluation of complete WLAN systems in a large reverberation chamber," IEEE Trans. Electromagn. Compat., vol. 55, no. 5, pp. 806-815, Oct. 2013.

[6] N. Arsalane, M. Mouhamadou, C. Decroze, D. Carsenat, M. A. GarciaFernandez, and T. Monediere, "3GPP channel model emulation with analysis of MIMO-LTE performances in reverberation chamber," Int. J. Antennas Propag., vol. 2012, no. Article ID 239420, p. 8, 2012.

[7] P.-S. Kildal, A. Hussain, X. Chen, C. Orlenius, A. Skårbratt, J. Åsberg, T. Svensson, and T. Eriksson, "Threshold receiver model for throughput of wireless devices with MIMO and frequency diversity measured in reverberation chamber," IEEE Antennas Wireless Propag. Lett, vol. 10, pp. 1201-1204, 2011.

[8] X. Chen, P.-S. Kildal, and M. Gustafsson, "Characterization of implemented algorithm for MIMO spatial multiplexing in reverberation chamber," IEEE Trans. Antennas Propag., vol. 61, no. 8, pp. 4400-4404, Aug. 2013.

[9] M. R. D. Rodrigues, I. Chatzigeorgiou, I. J. Wassell, and R. Carrasco, "Performance analysis of turbo codes in quasi-static fading channels," IET Commun., vol. 2, no. 3, pp. 449-461, 2008.

[10] R. Tian, B. K. Lau, and Z. Ying, "Multiplexing efficiency of MIMO antennas," IEEE Antennas Wireless Propag. Lett, vol. 10, pp. 183-186, 2011.

[11] N. Prasad and M. K. Varanasi, "Outage theorems for MIMO block-fading channels," IEEE Trans. Inf. Theory, vol. 58, no. 7, pp. 2159-2168, Jul. 2006

[12] LTE Physical Layer - General Description 3rd Generation Partnership Project, 2007, Tech. Rep. TS 36201, V8.1.0.

[13] Z. Kang, K. Yao, and F. Lorenzelli, "Nakagami-m fading modeling in the frequency domain for OFDM system analysis," IEEE Commun. Lett., vol. 7, no. 10, pp. 484-486, Oct. 2003.

[14] J. G. Kostas and B. Boverie, "Statistical model for a mode-stirred chamber," IEEE Trans. Electromagn. Compat., vol. 33, no. 4, pp. 366-370, Nov. 1991.

[15] A. Paulraj, R. Nabar, and D. Gore, Introduction to Space-Time Wireless Communication. Cambridge, U.K.: Cambridge Univ. Press, 2003.

[16] X. Chen, "Spatial correlation and ergodic capacity of MIMO channel in reverberation chamber," Int. J. Antennas Propag., vol. 2012, no. Article ID 939104, p. 7, 2012.

[17] W. Weichselberger, M. Herdin, H. Ozcelik, and E. Bonek, "A stochastic MIMO channel model with joint correlation of both link ends," IEEE Trans. Wireless Commun., vol. 5, no. 1, pp. 90-100, Jan. 2006

[18] X. Chen, P.-S. Kildal, J. Carlsson, and J. Yang, "MRC diversity and MIMO capacity evaluations of multi-port antennas using reverberation chamber and anechoic chamber," IEEE Trans. Antennas Propag, vol. 61, no. 2, pp. 917-926, Feb. 2013.

[19] IEEE 802 Part 16: Air Interface for Broadband Wireless Access Systems IEEE 802.16 Working Group, 2007.

[20] G. Berardinelli et al., "On the feasibility of precoded single user MIMO for LTE-A uplink," J. Commun., vol. 4, no. 3, pp. 155-163, Apr. 2009.

[21] T. B. Hansen, "Correlation and capacity calculations with reference antennas in an isotropic environment," Int. J. Antennas Propag., vol. 2012, no. Article ID 540649, p. 14, 2012.

[22] I. Szini, G. F. Pedersen, A. Scannavini, and L. J. Foged, "MIMO $2 \times 2$ reference antennas concept," presented at the Eur. Conf. Antennas and Propag. (EuCAP), Prague, Czech, Mar. 26-30, 2012.

[23] X. Chen and P.-S. Kildal, "Theoretical derivation and measurements of the relationship between coherence bandwidth and RMS delay spread in reverberation chamber," presented at the Eur. Conf. Antennas and Propag. (EuCAP), Mar. 23-27, 2009.

[24] O. Delangre, P. D. Doncker, M. Lienard, and P. Degauque, "Delay spread and coherence bandwidth in reverberation chamber," Electron. Lett., vol. 44, no. 5, Feb. 2008. 
[25] P. Jung and J. J. Blanz, "Joint detection with coherent receiver antenna diversity in CDMA mobile radio systems," IEEE Trans Veh. Technol., vol. 44, no. 1, pp. 76-88, Feb. 1995.

[26] G. J. M. Janssen, P. A. Stigter, and R. Prasad, "Wideband indoor channel measurements and BER analysis of frequency selective multipath channels at 2.4, 4.75, and $11.5 \mathrm{GHz}$," IEEE Trans. Commun., vol. 44, no. 10, pp. 1272-1288, Oct. 1996.

[27] S. Wyne, K. Haneda, S. Ranvier, F. Tufvesson, and A. F. Molisch, "Beamforming effects on measured mm-wave channel characteristics," IEEE Trans. Wireless Commun., vol. 10, pp. 3553-3559, Nov. 2011.

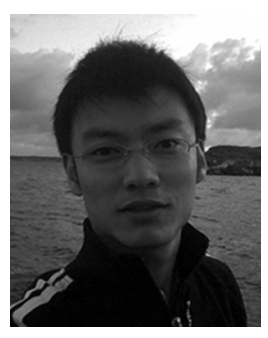

Xiaoming Chen received the B.Sc. degree in electrical engineering from Northwestern Polytechnical University, Xi'an, China, in 2006, and M.Sc. and $\mathrm{Ph} . \mathrm{D}$. degrees in electrical engineering from Chalmers University of Technology, Gothenburg, Sweden, in 2007 and 2012, respectively.

$\mathrm{He}$ is currently a Postdoctoral Researcher in the Department of Signals and Systems, Chalmers University of Technology. His current research areas include reverberation chamber measurements, multi-antenna channel characterization, OTA testing, and statistical electromagnetics. He is a reviewer of six IEEE journals. 